\title{
A Controversial Case of a Double Hepatic Incidentaloma in a Male Patient with Kidney Failure
}

\author{
Luca Rinaldi ${ }^{1^{*}}$, Claudia Altobelli ${ }^{2}$, Ilario De $\mathrm{Sio}^{3}$, Pina Acampora ${ }^{2}$, Maria Luisa Abategiovanni ${ }^{2}$, \\ Miriam Zacchia ${ }^{2}$, Francesco Trepiccione ${ }^{2}$, Stefania Milione ${ }^{1}$, Luigi Maria Vitale ${ }^{3}$, Pietro \\ Anastasio $^{2}$ and Giovambattista Capasso ${ }^{2}$ \\ ${ }^{1}$ Department of Medical, Surgical, Neurological, Metabolic and Geriatric Sciences, Università della Campania "Luigi \\ Vanvitelli", Italy \\ ${ }^{2}$ Section of Nephrology, Department of Cardiothoracic and Respiratory Sciences, Università della Campania "Luigi \\ Vanvitelli", Italy \\ ${ }^{3}$ Divisions of Hepatogastroenterology, Department of Clinical and Experimental Medicine, Università della \\ Campania "Luigi Vanvitelli", Italy
}

Received: February 7, 2017; Accepted: May 3, 2017; Published: May 15, 2017

*Corresponding author:Luca Rinaldi, Department of Medical, Surgical, Neurological, Metabolic and Geriatric Sciences, Università della Campania "Luigi Vanvitelli", Piazza L. Miraglia , 80100 Naples, Italy, Tel: +39 0815665081; Fax: +39 0815665080; E-mail: lucarinaldi@hotmail.it

\section{Abstract}

Hepatic incidentalomas are incidentally lesions detected during investigations for other pathologies. We describe a pre-dialysis kidney failure male patient case. During abdominal ultrasound exam, two hypoechoic lesions were found. The non-univocal results of contrast enhancement ultrasound and magnetic resonance suggested the diagnosis of hepatic adenoma, but required further examinations. The liver biopsy showed cellular atypia compatible with both hepatic adenoma and differentiated hepatocellular carcinoma. Transient elastography indicated the absence of significant fibrosis. According to this data, we have leant towards benign hepatic lesions, because the presence of hepatocarcinoma in a non-cirrhotic liver is unlikely to be verified.

Keywords: Hepatocellular Adenoma; Hepatic Incidentaloma; Contrast Enhancement Ultrasound; Transient Elastography; Hepatocellular Carcinoma.

\section{Abbreviations}

HCC: Hepatocellular Carcinoma; HCA: Hepatocellular Adenoma; BMI: Body Mass Index;

K-DIGO: Kidney Disease- Improving Global Outcomes; CEUS: Contrast Enhancement Ultrasonography; MRI: Magnetic Resonance Imaging; IVC: Inferior Vena Cava; CT: Computed Tomography scan

\section{Introduction}

The liver incidentaloma is increasingly found in asymptomatic patients during investigations for other pathologies [1]. The main hepatic solid lesions can be distinguished into benign lesions (including angioma, focal nodular hyperplasia and adenoma) and malignants, like Hepatocellular Carcinoma (HCC). In some cases, it is not easy to differentiate the two types of lesion.

Benign hepatic lesions are found in $20-50 \%$ of cases [23]. The Hepatocellular Adenoma (HCA) is a rare benign tumor that develops in non-cirrhotic patients. There is a known link with the use of oral contraceptives in women or glycogen storage [4]; HCA in male is rare; it is also associated with metabolic syndrome or anabolic treatments [5-6]. Usually, it's a single and variable size mass located in the right lobe in the subcapsular. Histologically, it's characterized by the loss of normal lobular hepatic architecture cords of normal or slightly atypical hepatocytes, with high glycogen and fat content in absence of bile ducts and Kupffer cells and often intratumoral haemorrhage [2].

At ultrasound, the adenoma appears as an isohypoechoic lesion; the Doppler shows a peculiar abundant peripheral vasculature, arterial and venous, and intratumoral vascularity of venous type [7]. It is also reported the possibility of a malignant lesion transformation with a variable incidence; this possibility seems to be associated with the subtype of mutate $\beta$-catenin or a large size of lesion [8-9]. However, some features contrastographic detectable by diagnostic tests, making it sometimes difficult to distinguish from well-differentiated HCC [10].

\section{Case report}

A 33-years-old Italian man, with a Body Mass Index (BMI) of $26 \mathrm{Kg} / \mathrm{m}^{2}$, came to our observation for nephrologic follow-up.

The patient is suffering from membranoproliferative glomerulonephritis type I, diagnosed when he was 20 by kidney needle biopsy. He is currently in pre-dialysis for a chronic kidney disease stage $\mathrm{V}$, according to Kidney Disease- Improving global 
outcomes (K/DIGO).

During hospitalization, the patient underwent an abdomen ultrasound, that incidentally detected two liveroccupying lesions on the II (hypoechoic) and VIII segment (isoechoic with hypoechoic flange), respectively of $47 \mathrm{~mm}$ and 69 $\mathrm{mm}$ (Figure 1). Routine laboratory investigations were normal. The liver function tests were within the normal range, with the exception of GGT (>150 U/L). Serum hepatitis viral markers were negative. Tumor markers, including carcinoembryonic antigen, $\alpha$-feto-protein and carbohydrate antigen 19-9, were in the normal range.

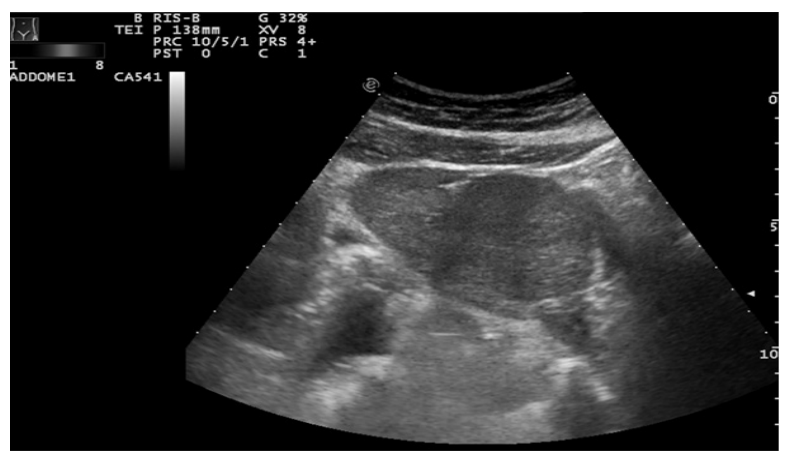

Figure 1a

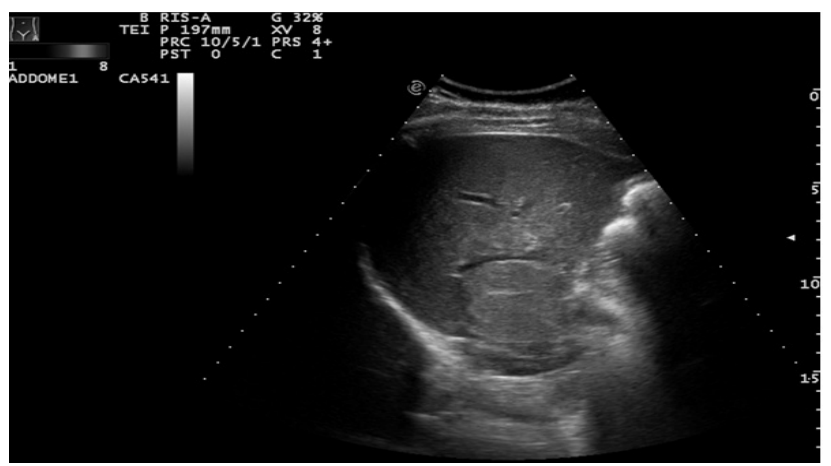

Figure 1b

Figure 1: Hypoechoic lesion on the II hepatic segment, diameter $47 \mathrm{~mm}$ (1a); isoechoic with hypoechoic flange lesion on the VIII segment, diameter $69 \mathrm{~mm}(1 \mathrm{~b})$.

WeperformedaContrastEnhancementUltrasonography (CEUS) with sulphur hexafluoride microbubbles (SonoVue ${ }^{\circledR}$, Bracco, and Milan, Italy) to better investigate the features of those lesions. An early arterial enhancement (15-20 seconds) was found, with a portal wash out of about 80-90 seconds for the VIII segment lesion, and a late wash out ( $>120$ seconds) for the II segment lesion (Figure 2-3). The vascular dynamics suggested the presence of two primitive hepatic lesions, probably benign, compatible with adenoma. To better address the nature of the lesions the patient underwent to a percutaneous liver biopsy. So, with a $16 \mathrm{~mm}$ needle (modified Menghini needle ${ }^{\circledR}$, Biomedical) we proceeded with an ultrasound-guided biopsy of the II segment lesion. Unfortunately, the VIII segment lesion was poorly accessible for the biopsy. The histological analysis demonstrated the presence of a well differentiated liver neoplasia. The tumor cells expressed the following immunophenotypes: CK7, CK20, CEA, CD34, glypican. The $\beta$-catenin signal was not nuclear achievement. However, the report concluded that it was not possible to differentiate between adenoma and welldifferentiated HCC and suggested to match clinical and laboratory data.

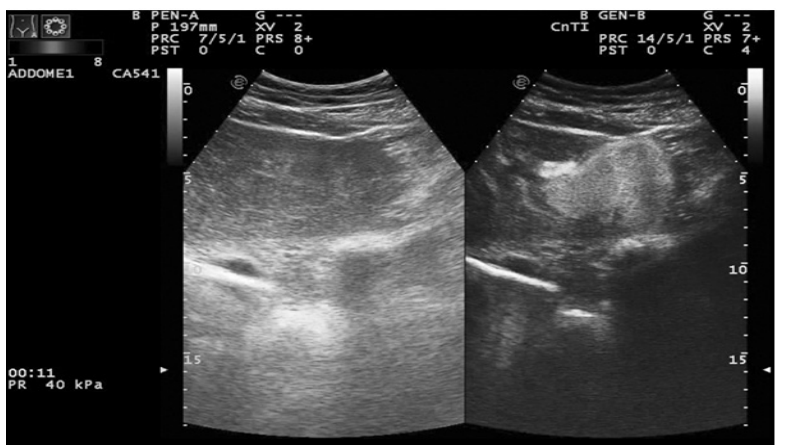

Figure 2a

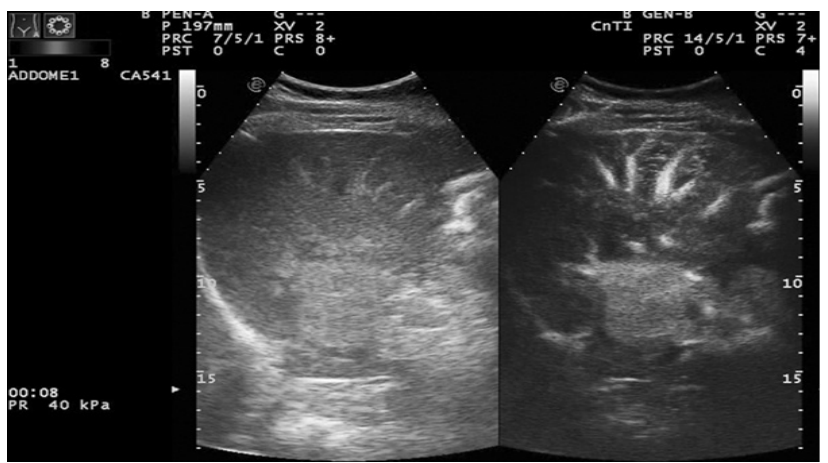

Figure 2b

Figure 2: Early arterial enhancement (06-15 seconds), in the II segment lesion (2a), and VIII segment lesion (2b)

Due to the inconclusiveness of the hystological report, a second level imaging test was performed. Given the impossibility to administer gadolinium, because of the severe renal insufficiency, the patient underwent the Magnetic Resonance (MRI) without contrast agent. MRI report described a normal volume liver with homogeneous signal intensity and the presence of two coarse expansive formations, one of which localized to the II-III segments (64 x $43 \mathrm{~mm}$ ) in partial exophytic development from the profile of the organ, while the other localized at the VIIIVII segment ( $66 \times 44 \mathrm{~mm}$ ) in close proximity to the right branch of the portal vein and inferior vena cava (IVC) compression. These formations showed a hyperintense signal intensity on T2 and hypointense on T1 (Figure 4). It should be noted that the formation of the VIII-VII segment showed signal characteristics suggestive of increased necrotic component. 


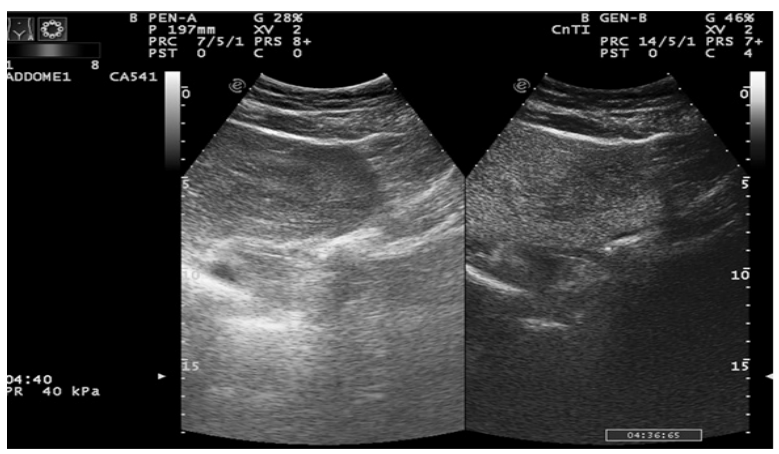

Figure 3a

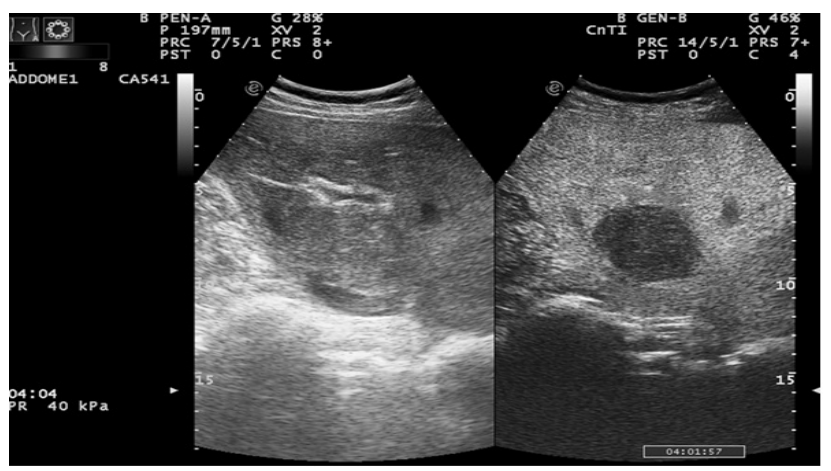

Figure 3b

Figure 3: Portal wash out of the II segment lesion (3a) and VIII segment lesion (3b)

To get a rough estimate of the level of fibrosis, a noninvasive measurement of the liver stiffness was conducted using the Fibroscan ${ }^{\circledR}$ device. Transient elatography showed liver stiffness of $6.5 \mathrm{Kpa}$, compatible with the absence of significant fibrosis ( $<$ F2 Metavir). It was required specialist expertise of the hepatologist who advised counseling c/o Transplant Center to evaluate the opportunity of a double transplant (liver/kidney); or, alternatively, the possibility of surgical resection and control of chronic renal failure with dialysis treatment. The patient refused liver/kidney transplant and we performed a monitoring of hepatic lesions. After one year there was no change in the number and size of both lesions.

\section{Discussion}

The hepatic adenoma is a typical lesion of the females linked to the acceptance of oral contraceptives; rarely, it occurs in males and it appears linked to steroid use, obesity or glycogen storage disease. The singularity of our case is represented by the fact that the patient had none of these predisposing factors. In particular, we noted the absence of significant metabolic defects (hyperlipidemia, increased CPK, fasting blood glucose, uric acid alterations). Moreover, the presence of advanced renal failure has placed the diagnostic deepening problems. In fact, it was not possible to do Computed Tomography (CT) scan or MRI with

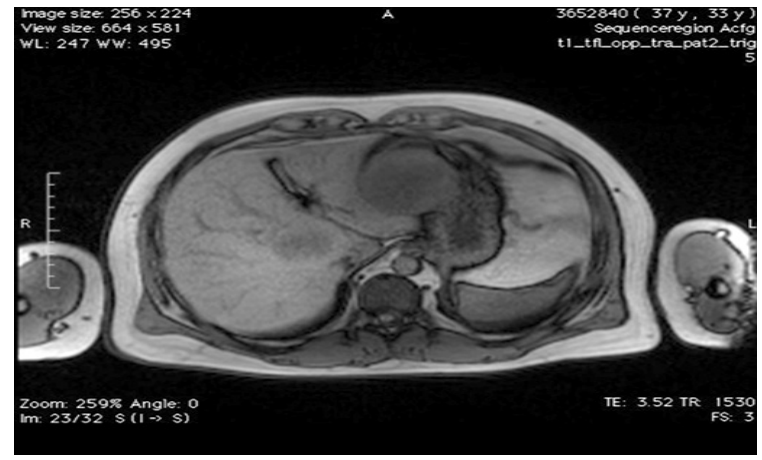

Figure 4a

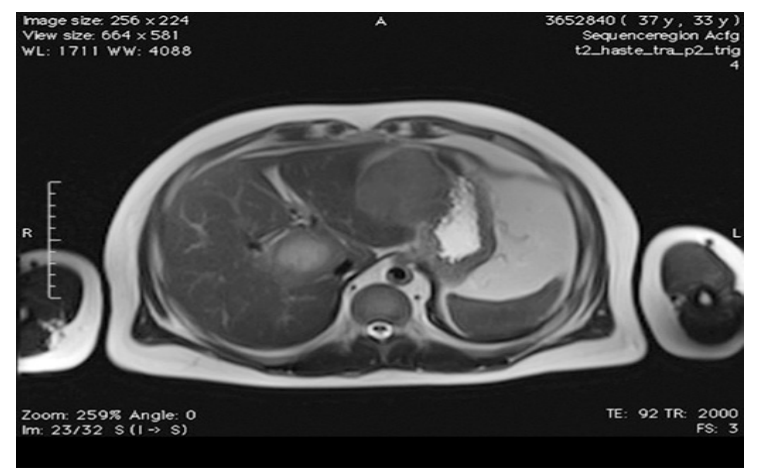

Figure 4b

Figure 4: MRI of the II segment lesion (4a) and VIII segment lesion (4b)

contrast agents because of renal failure and only one of the two lesions allowed safe access for liver biopsy.

CEUS showed two lesions with early hypervascularization $(15-20 \mathrm{sec})$ and different timing in the wash-out: namely the lesion of the second segment showed a wash-out around 80 seconds, while that of the seventh 120 seconds. Some studies have placed focus on the timing of the wash out to differentiate the HCC from other malignant lesions (such as cholangiocarcinoma, lymphoma); in particular, an earlier wash-out $(<55 \mathrm{sec}$ ) has been associated with malignancy not due to HCC [11]; in addition, it has been shown that HCA have a a rapid and homogeneous enhancement and showed a slow wash-out pattern in the arterial phase on CEUS, with subcapsular tortuous arteries [12-13]. Nevertheless, the HCA and also the focal nodular hyperplasia may have similar characteristics to a well differentiated HCC at all stages of CEUS and a late wash-out may be more an expression of a well-differentiated HCC [14]. The biopsy of the lesion was unfortunately not conclusive, as the histological analysis did not allow addressing whether the lesion was and adenoma or an HCC thus.

HCC usually arises on a cirrhotic liver or with a fair degree of fibrosis in chronic liver disease. For this reason, we proceeded with transient elastography to estimate fibrosis; 
the liver stiffness value of less than 7 kilopascals rules out the presence of significant fibrosis, especially cirrhosis. This procedure is now considered of high accuracy in the detection of cirrhosis and severe fibrosis [15-16].

The exam demonstrated that the two lesions were developed in a substantially healthy liver. The follow up of the patients revealed that the lesions did not change in number and size; that no deterioration of liver function occurred, supporting the hypothesis that both nodules were benign liver lesions.

The management of a patient with dual HCA is based on the assessment of the risk of complications. In particular, three studies of patients undergoing to surgery for adenoma, found a low but significant percentage of malignance. This event was associated with male gender associated with metabolic syndrome, the mutation of $\beta$-catenin and the increase of alpha-fetoprotein. Instead, complications, such as hemorrhage, appeared related to the size $(>5 \mathrm{~cm})$, and to teleangiectatic hepatocellular adenoma, a subcategory of inflammatory HCA [17-20]. In accordance with the American College of Gastroenterology Clinical Guideline for diagnosis and management of focal liver lesions, we decided to follow potential changes of the lesions over the months, as the patient presented high risk of complications for the size (bleeding) and low risk of malignancy for the male gender, and the low expression of $\beta$-catenin [21].

The patient, also in consideration of its will, did not undergo surgery and is still monitored with ultrasound.

The histological sample allowed evaluating the immunohistochemical characteristics, and genetic molecular expression must enable to identify the subtype of HCA and provide an optimal clinical management [22]. Nevertheless, as in our case, the histologic sample could provide atypical aspects difficult to be interpreted. The II level radiological finding like the CEUS ensures a good accuracy similar to CT to characterize the focal liver lesion, as showed in several papers [23-24].

\section{Conclusion}

By now, the importance of non-invasive procedures (Transient elastography and ultrasound) has been recognized. These are used as supportive techniques or as alternative to second and third level instrumental techniques in the initial evaluation of the patient and in monitoring over time. In our case, the HCA diagnosis remained controversial, and the patient's management is focused on the short-distance clinical monitoring.

\section{References}

1. Jonathan B. Koea Hepatic incidentaloma: the rule of tens. HPB (Oxford). 2013;15(5):379-383. doi: 10.1111/j.1477-2574.2012.00595.x

2. Cristiano A, Dietrich A, Spina JC, Ardiles V, de Santibañes E. Focal nodular hyperplasia and hepatic adenoma: current diagnosis and management. Updates Surg. 2014;66(1):9-21. doi: 10.1007/s13304013-0222-3

3. Kaltenbach TE, Engler P, Kratzer W, Oeztuerk S, Seufferlein T, Haenle
$\mathrm{MM}$, et al. Prevalence of benign focal liver lesions: ultrasound investigation of 45,319 hospital patients. Abdom Radiol (NY). 2016;41(1):25-32. doi: 10.1007/s00261-015-0605-7

4. Pulpeiro JR, Orduna M, Jimenez J, Gallego SM, Millan JM. Primary hepatocellular adenoma in men. J Clin Ultrasound. 1989;17(4):269274.

5. Rosencrantz RA, Wu Y, Sonke PY, Yusuf Y. Giant hepatocellular adenoma in a previously obese thirteen-year-old boy. Ann Hepatol. 2015;14(4):559-563.

6. Bunchorntavakul C, Bahirwani R, Drazek D, Soulen MC, Siegelman ES, Furth EE, et al. Clinical features and natural history of hepatocellular adenomas: the impact of obesity. Aliment Pharmacol Ther. 2011;34(6):664-674. doi: 10.1111/j.1365-2036.2011.04772.x

7. Kong WT, Wang WP, Huang BJ, Ding H, Mao F, Si Q. Contrast-enhanced ultrasound in combination with color Doppler ultrasound can improve the diagnostic performance of focal nodular hyperplasia and hepatocellular adenoma. Ultrasound Med Biol. 2015;41(4):944-951. doi: 10.1016/j.ultrasmedbio.2014.11.012

8. Foster JH, Berman MM. The malignant transformation of liver cell adenomas. Arch Surg. 1994;129(7):712-717.

9. Kim GJ, Seok JY, Rhee H, Choi JY, Choi JS, Kim KS, et al. $\beta$-Catenin Activated Hepatocellular Adenoma: A report of three cases in Korea. Gut Liver. 2014;8(4):452-458. doi: 10.5009/gnl.2014.8.4.452

10. Brancatelli G, Federle MP, Vullierme MP, Lagalla R, Midiri M, Vilgrain V. CT and MR imaging evaluation of hepatic adenoma. J Comput Assist Tomogr. 2006;30(5):745-750.

11. de Sio I, Iadevaia MD, Vitale LM, Niosi M, Del Prete A, de Sio C, et al. Optimized contrast-enhanced ultrasonography for characterization of focal liver lesions in cirrhosis: A single-center retrospective study. United European Gastroenterol J 2014;2(4):279-287. doi: $10.1177 / 2050640614538964$

12. Sutherland T, Temple F, Lee WK, Hennessy O. Evaluation of focal hepatic lesions with ultrasound contrast agents. J Clin Ultrasound. 2011;39(7):399-407. doi: 10.1002/jcu.20847

13. Dong Y, Zhu Z, Wang WP, Mao F, Ji ZB. Ultrasound features of hepatocellular adenoma and the additional value of contrast-enhanced ultrasound. Hepatobiliary Pancreat Dis Int. 2016;15(1):48-54.

14. Zhu XL, Chen P, Guo H, Zhang N, Hou WJ, Li XY, et al. Contrast-Enhanced Ultrasound for the Diagnosis of Hepatic Adenoma. J Int Med Res. 2011;39(3):920-928.

15. Rinaldi L, Restivo L, Guerrera B, et al. Hepatic Steatosis And NecroInflammatory Activity Overestimate Liver Stiffness by Transient Elastography in Staging Liver Fibrosis in Chronic Hepatitis C. J Hepat Res. 2014;1(3):1-6.

16. Tsochatzis EA, Gurusamy KS, Ntaoula S, Cholongitas E, Davidson BR, Burroughs AK. Elastography for the diagnosis of severity of fibrosis in chronic liver disease: a meta-analysis of diagnostic accuracy. J Hepatol. 


\section{1;54(4):650-659. doi: 10.1016/j.jhep.2010.07.033}

17. Dokmak S1, Paradis V, Vilgrain V, Sauvanet A, Farges O, Valla D, et al. A single-center surgical experience of 122 patients with single and multiple hepatocellular adenomas. Gastroenterology. 2009;137(5):1698-1705. doi: 10.1053/j.gastro.2009.07.061

18. An SL, Wang LM, Rong WQ, Wu F, Sun W, Yu WB, et al. Hepatocellular adenoma with malignant transformation in male patients with noncirrhotic livers. Clin J Cancer 2015; 24;34(5):217-224. doi: 10.1186/ s40880-015-0014-x

19. Stoot JH, Coelen RJ, De Jong MC, Dejong CH. Malignant transformation of hepatocellular adenomas into hepatocellular carcinomas: a systematic review including more than 1600 adenoma cases. HPB (Oxford). 2010;12(8):509-522. doi: 10.1111/j.1477-2574.2010.00222.x

20. Belghiti J, Cauchy F, Paradis V, Vilgrain V. Diagnosis and management of solid benign liver lesions. Nat Rev Gastroenterol Hepatol. 2014;11(12):737-749. doi: 10.1038/nrgastro.2014.151
21. Marrero JA, Ahn J, Rajender Reddy K, Americal College of Gastroenterology. ACG Clinical Guideline: The Diagnosis and Management of Focal Liver Lesions. Am J Gastroenterol. 2014;109(9):1328-1347; quiz 1348. doi: 10.1038/ajg.2014.213

22. Dhingra S, Fiel MI. Update on the New Classification of Hepatic Adenomas: Clinical, Molecular, and Pathologic Characteristics. Arch Pathol Lab Med. 2014;138(8):1090-1097. doi: 10.5858/arpa.20130183-RA

23. D’Onofrio M, Rozzanigo U, Masinielli BM, Caffarri S, Zogno A, Malagò R, et al. Hypoechoic Focal Liver Lesions: characterization with contrast enhanced Ultrasonography. J Clin Ultrasound 2005;33(4):164-172.

24. Li R, Guo Y, Hua X, He Y, Ding J, Guo A, et al. Characterization of Focal Liver Lesions: Comparison of Pulse-Inversion Harmonic ContrastEnhanced Sonography with Contrast-Enhanced . J Clin Ultrasound. 2007;35(3):109-117. 